\title{
Far-infrared vibrational spectra of all-trans, 9-cis and 13-cis retinal measured by $\mathrm{THz}$ time-domain spectroscopy
}

\author{
M. Walther, B. Fischer, M. Schall, H. Helm, P. Uhd Jepsen * \\ Fakultät für Physik, Albert-Ludwigs-Universität Freiburg, Stefan-Meier-Str. 19, D-79104 Freiburg, Germany
}

Received 14 August 2000; in final form 18 October 2000

\begin{abstract}
Low-frequency torsional vibration modes of the conjugated polyene chain of the biologically important chromophore retinal are of major relevance when studying its photoisomerization dynamics. We present absorptivity and dispersion spectra of three different retinal isomers in the far-infrared region between 10 and $100 \mathrm{~cm}^{-1}(0.3-3.0 \mathrm{THz})$ measured by terahertz time-domain spectroscopy at 298 and $10 \mathrm{~K}$. At low temperatures the observed broad absorption bands resolve into narrow peaks correlating to torsional modes of the molecule. Comparison of the absorption spectra of different retinal isomers enables the approximate localization of the modes within the molecule. Experimental linewidths and transition strengths are reported. (c) 2000 Elsevier Science B.V. All rights reserved.
\end{abstract}

\section{Introduction}

Protein-bound retinal molecules are the chromophores in the photoactive proteins rhodopsin and bacteriorhodopsin. Photoinduced isomerization of the chromophore molecule is the primary step in their photocycles. In rhodopsin the conformation change 11-cis $\rightarrow$ all-trans triggers the primary step of the vision process and the alltrans $\rightarrow 13$-cis isomerization is the initial step of the photosynthesis cycle in bacteriorhodopsin [1]. The reaction coordinate of this process is a welldefined torsion along one of the $\mathrm{C}=\mathrm{C}$ double bonds of the polyene chain. Conformational changes induced by photoexcitation of the conju-

\footnotetext{
${ }^{*}$ Corresponding author.

E-mail addresses: helm@uni-freiburg.de, http://frhewww. physik.uni-freiburg.de/terahertz/ (H. Helm), jepsen@uni-freiburg.de (P.U. Jepsen).
}

gated $\pi$-electron system of the linear $\mathrm{C}$-chain is the subject of extensive experimental and theoretical studies [2-4]. Optical and near-infrared $(<900 \mathrm{~nm})$ pump-probe techniques, where a femtosecond laser pulse initiates the isomerization process before a subsequent probe pulse monitors changes in the absorption characteristics of the sample, are currently the most effective way to investigate the dynamics of the photoisomerization. Transient absorption in the visible probes the electronic configuration and is therefore only an indirect probe for the isomeric state of the chromophore. These time-resolved experiments revealed that the 11-cis $\rightarrow$ all-trans isomerization in rhodopsin takes place within only $200 \mathrm{fs}$ [5]. Absorption in the mid-infrared 'finger-print' region between 1100 and $1300 \mathrm{~cm}^{-1}$ where $\mathrm{CCH}$ bending and $\mathrm{C}-\mathrm{C}$ stretching modes are active, has also been demonstrated to be sensitive to the isomeric configuration of retinals [6] albeit without time resolution. 
The observation of low-frequency delocalized vibrational modes offers direct insight into conformational dynamics of large molecules. The resonance frequencies of these modes are located in the far-infrared (FIR) part of the spectrum below $200 \mathrm{~cm}^{-1}$, typical for torsions in polyenes [7].

Damped oscillatory features in the visible $a b-$ sorption with a period of $550 \mathrm{fs}$, corresponding to a frequency of $60 \mathrm{~cm}^{-1}$, have been observed in absorption at $500 \mathrm{~nm}$ after isomerization and have been interpreted as a consequence of vibrational coherence [2,3] and as nonadiabatic wave packet motion on coupled potential energy surfaces [4]. This also indicates the relevance of FIR spectroscopy for a comprehensive understanding of the isomerization process.

Due to considerable experimental difficulties and the complexity of theoretical models only few experimental or theoretical investigations in the FIR spectral region are known $[8,9]$. $\mathrm{THz}$ timedomain spectroscopy (THz-TDS) [10] has been demonstrated to be a versatile tool for spectroscopy on a wide variety of different samples in the FIR and recently this new technique was applied to examine DNA and other biomolecules [11]. THz-TDS relies on coherent generation and detection of an ultrashort probe pulse with a correspondingly large bandwidth covering the spectral region from 0.1 to $4 \mathrm{THz}$. The duration of the $\mathrm{THz}$ pulse is less than 1 ps enabling transient FIR spectroscopy with time resolution well into the femtosecond regime [12-14]. A realistic future goal which we are pursuing is therefore to observe the molecular dynamics directly by studying the lowfrequency modes during the isomerization process of retinal in a time-resolved experiment.

In this work we measure and analyze the FIR absorption and dispersion of three retinal isomers that occur in the photoactive proteins rhodopsin, bacteriorhodopsin, isorhodopsin or their photoproducts, namely, all-trans, 9-cis and 13-cis retinal. The three isomers are shown in Fig. 1. Our observation of substantial differences between the low-frequency vibrational spectra of the three isomers demonstrates the feasibility of time-resolved experiments in the FIR using THzTDS. (a)

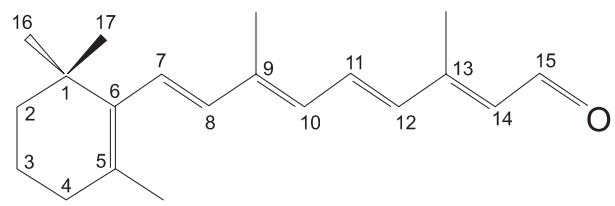

(b)

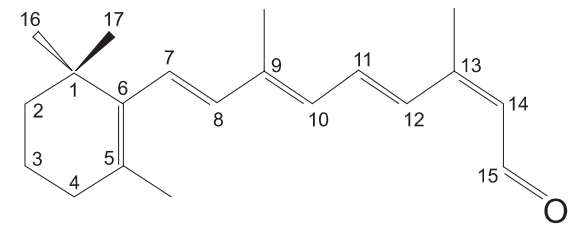

(c)

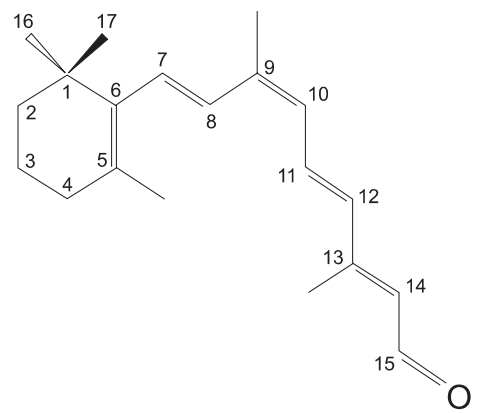

Fig. 1. Three different isomers of retinal: (a) all-trans; (b) 13-cis; (c) 9-cis configuration.

\section{Methods and materials}

All-trans and 9-cis retinal were purchased from Sigma-Aldrich and 13-cis retinal from ICN Biomedicals and used without further purification. All samples were stored in the dark below $0^{\circ} \mathrm{C}$. Samples were prepared by mixing retinal with polyethylene (PE) powder at a mass ratio of 1:1 and pressing the mixture to disks of thicknesses between 270 and $900 \mu \mathrm{m}$ applying an approximate pressure of $400 \mathrm{~kg}$ $\mathrm{cm}^{-1}$. Since PE is nearly transparent in the FIR it is a suitable filling material for spectroscopic applications in this spectral region.

We use a standard $\mathrm{THz}$ spectrometer setup $[10,15]$ as illustrated in Fig. 2. Pulses of $40 \mathrm{fs}$ duration from a Ti:sapphire oscillator are used to drive two photoconductive antennas, one which emits short pulses of $\mathrm{THz}$ radiation, the other, acting as a gated detector, monitors the temporal 


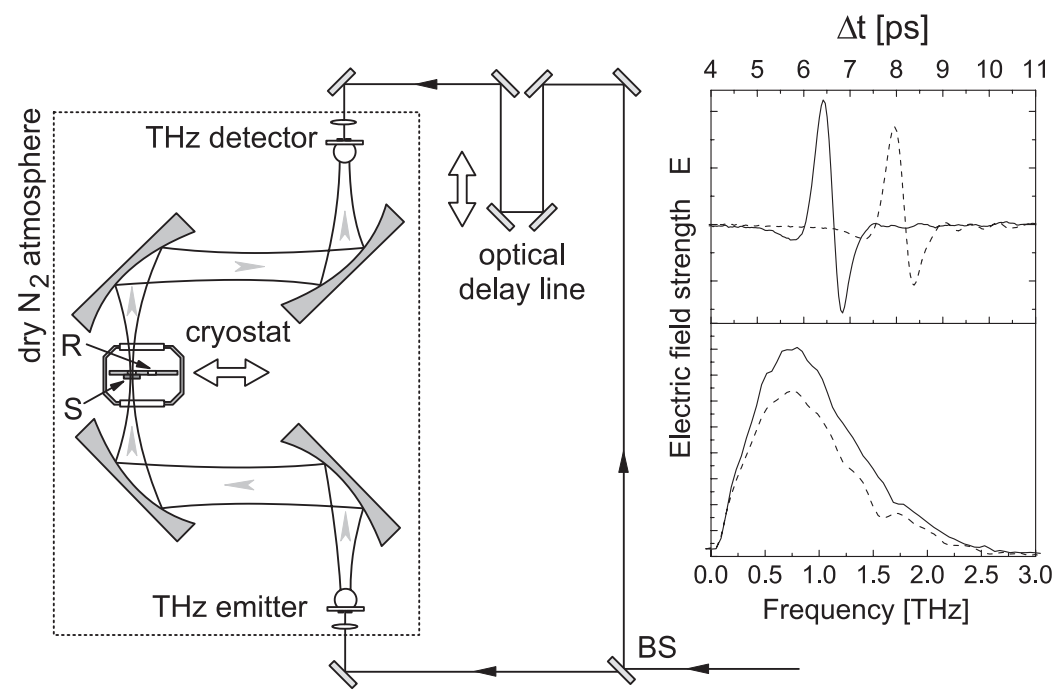

Fig. 2. THz spectrometer setup with the cryostat, containing reference (R) and sample aperture (S), mounted in the THz beam path. The inset shows a typical THz pulse and the corresponding frequency spectrum before and after passing the 9-cis retinal sample.

profile of the THz electric field strength. The first two paraboloidal mirrors in the $\mathrm{THz}$ beam path collimate and focus the $\mathrm{THz}$ beam to a frequencyindependent spotsize of $2 \mathrm{~mm}$ at the position of the sample [16]. The sample disk is mounted in a cryostat equipped with Teflon windows, transparent for THz-radiation. The temperature is measured near the sample with a calibrated Si-diode. The cryostat can be moved so that the $\mathrm{THz}$ beam passes through either the sample or through an empty aperture with the identical size as the clear aperture of the sample. The two last paraboloidal mirrors recollimate and focus the $\mathrm{THz}$ beam onto the detector. The whole system is placed in a closed box purged with dry nitrogen in order to eliminate absorption by water vapor in the beam path. The setup has a useful bandwidth from $100 \mathrm{GHz}$ to $2.9 \mathrm{THz}\left(6-100 \mathrm{~cm}^{-1}\right)$. The inset in Fig. 2 shows a typical $\mathrm{THz}$ pulse and its corresponding frequency spectrum before and after propagation through the 9-cis retinal sample.

\section{Results and discussion}

The field of a $\mathrm{THz}$ pulse transmitted through a sample is modified by dispersion and absorption of the substrate. The ratio of the electrical field strengths before $E_{\mathrm{r}}$ and after transmission $E_{\mathrm{s}}$ is given by

$E_{\mathrm{s}} / E_{\mathrm{r}}=T(n) \exp \{-\alpha d+i n \omega d / c\}$,

where $d$ is the thickness of the sample, $\omega$ the frequency of the radiation, $c$ the speed of light in vacuum and $T(n)$ is a factor which accounts for reflection losses at the sample surfaces. Both the refractive index $n$ and the absorption coefficient $\alpha$ can be determined from the ratio of the measured THz fields. The molar absorptivity $\alpha_{M}$ is related to the absorption coefficient by $\alpha_{\mathrm{M}}=\alpha / K$, where $K$ is the sample concentration.

In Figs. 3-5 the frequency dependences of $\alpha_{M}$ and $n$ of all-trans, 9-cis and 13-cis retinal are shown at 298 and $10 \mathrm{~K}$. Comparison of spectra recorded through different sample thicknesses assured that the spectra are essentially free of features due to etalon effects. The PE filler has a constant refractive index of approximately 1.54, depending slightly on the applied pressure in the sample preparation. The absorption of PE is small $\left(<5 \mathrm{~cm}^{-1}\right.$ below $\left.3 \mathrm{THz}\right)$, and we subtracted this background from the absorption data presented here. The strong differences between the spectra of the different samples immediately show that FIR 


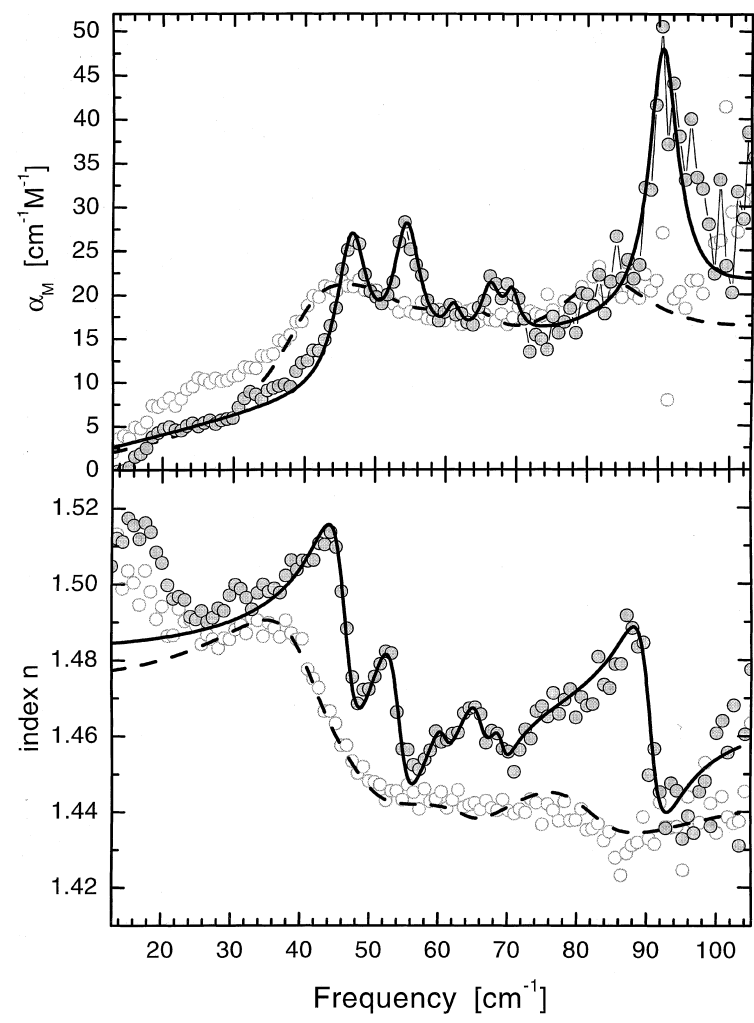

Fig. 3. Molar absorptivity and index of refraction of all-trans retinal in PE at 298 (open circle) and $10 \mathrm{~K}$ (closed circle). The solid line is a fit according to a sum of six Lorentzian oscillators fitted simultaneously to index and absorption data of the cooled sample. The dashed line is a fit to the room temperature data (see text).

absorption is an excellent probe of the isomeric structure of the retinal chromophore.

All samples show broad absorption features at room temperature resolving into several narrow resonances at low temperature. The resonances shift to slightly higher frequencies and their linewidths narrow at lower temperature. All absorption peaks are accompanied by a characteristic change of the refractive index.

Using the standard expression for Lorentzian oscillators it is possible to extract the center frequencies $v_{j}$, the linewidths $\Gamma_{j}$ and the oscillator strengths $S_{j}$ of the different modes. The Lorentzian line profile model does not take into account inhomogeneous broadening of the lines. In our experimental situation, where the sample is cold and pressed into a PE matrix, inhomogeneous broad-

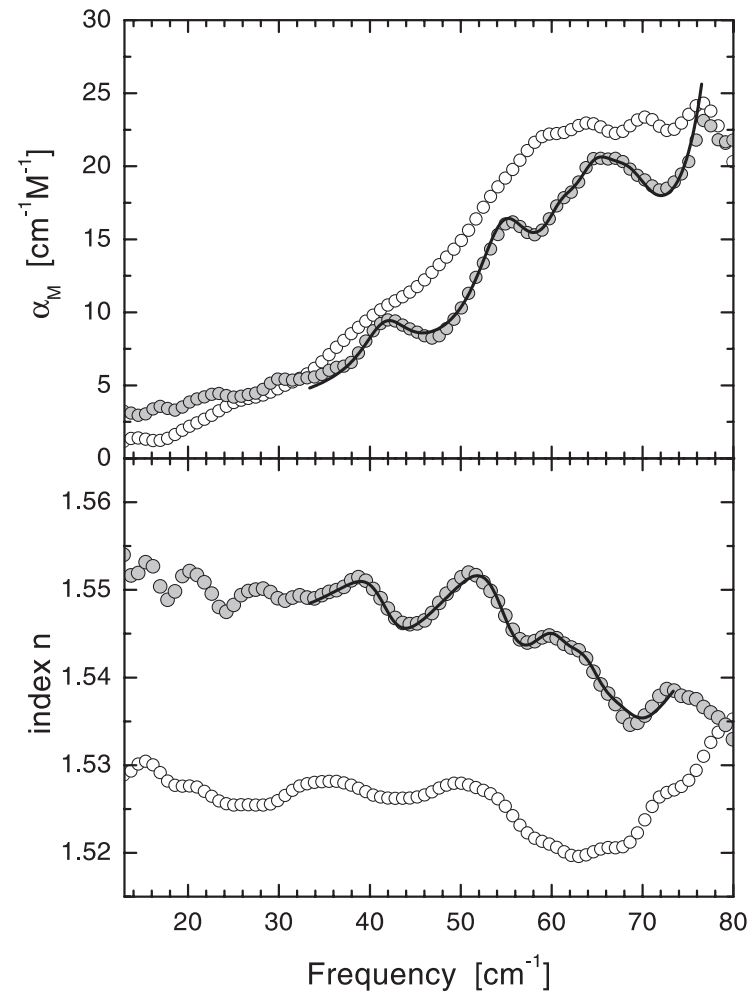

Fig. 4. Molar absorptivity and dispersion spectra of 13-cis retinal in PE at 298 (open circle) and $10 \mathrm{~K}$ (closed circle). A good fit between 38 and $74 \mathrm{~cm}^{-1}$ (solid line) was achieved by using the parameters given in Table 1 .

ening cannot be ruled out, and therefore a modified model with Gaussian or Voigt profiles or a Gaussian distribution of Lorentz profiles should in principle be more appropriate. Their main difference from a Lorentzian line profile is found in the wings, where in our case information is lost due to the close spacing of the lines and due to background absorption. We fitted the Lorentz model since it offers a simple description of the complex refractive index across the line profile. It is of crucial importance for the accuracy of the fitting procedure, that our method permits simultaneous determination of both absorption and index data. We used the frequency-dependent complex dielectric function [17]

$\epsilon(v)=\epsilon_{\infty}+\sum_{j} \frac{S_{j} v_{j}^{2}}{v_{j}^{2}-v^{2}-\mathrm{i} v \Gamma_{j}}=(n+\mathrm{i} \kappa)^{2}$, 


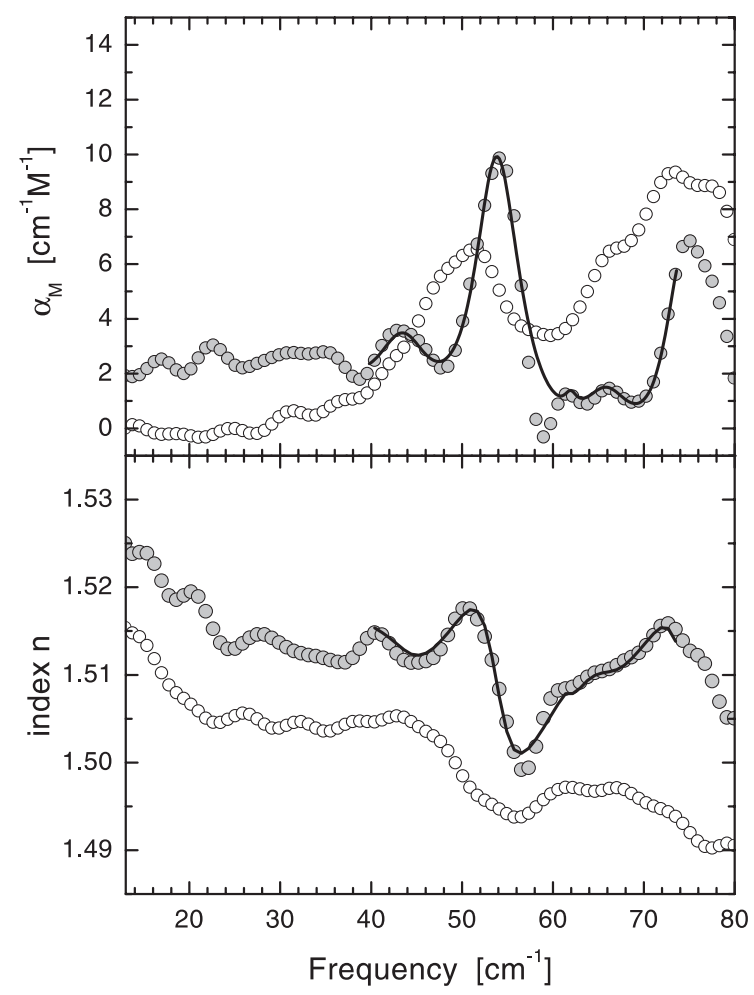

Fig. 5. Molar absorptivity and dispersion spectra of 9-cis retinal in PE at 298 (open circle) and $10 \mathrm{~K}$ (closed circle). The solid line is the fit based on parameters given in Table 1 (see also text).

where the sum is taken over the different oscillators. The high-frequency contribution to the dielectric function is denoted by $\epsilon_{\infty}$. The imaginary part of the complex index $\kappa$ is related to the absorption coefficient by $\kappa(v)=\alpha c / 4 \pi v$.

In Fig. 3 we demonstrate the suitability of this model for the data of the cooled all-trans isomer. We achieve a good simultaneous fit of the absorptivity and index from 25 to $105 \mathrm{~cm}^{-1}$ using six oscillators. The deviation from the data below $25 \mathrm{~cm}^{-1}$ is due to an artifact arising from the apertures used. In the fits of the absorptivity of alltrans and 13-cis we subtracted a linear background from the data. We attribute this background to impurities in the sample. The center frequencies, linewidths and oscillator strengths can be extracted from the fit, and the results are listed in Table 1. The errors given represent the standard deviations
(S.D.) of the fit. The line strengths carry an additional uncertainty of $\pm 4 \%$ due to uncertainty in the sample thickness. The absolute frequency scale was calibrated using the known positions of water vapor absorption lines.

From the oscillator strengths $S_{j}$ the band intensities $I_{j}$ in units of $\mathrm{km} \mathrm{mol}^{-1}$ can be evaluated by applying the conversion $I_{j}=\pi^{2} S_{j} v_{j}^{2} / n K$ where $v_{j}$ denotes the center frequencies in $\mathrm{cm}^{-1}$. The mean value of the refractive index $n$ around the resonance frequency can be used in the equation. The sample concentrations $K$ are $1.89 \mathrm{~mol} \mathrm{l}^{-1}$ for the all-trans and $1.13 \mathrm{~mol}^{-1}$ for the 9-cis and 13cis samples. The calculated band strengths are listed in Table 1.

Starting from the parameters of the $10 \mathrm{~K}$ alltrans-fit, we can match the index and absorptivity of the $298 \mathrm{~K}$ measurement very well by red-shifting all center frequencies by $4.5 \mathrm{~cm}^{-1}$, broadening the linewidths by a factor of approximately 2 , and adjusting the oscillator strengths. A detailed study of the temperature dependence of the individual modes is currently being undertaken.

On the basis of the parameters for the all-trans configuration we also modeled the spectra of the 9-cis and 13-cis isomers in Figs. 4 and 5 in the spectral region from 40 to $75 \mathrm{~cm}^{-1}$. In the 9-cis case additional modes centered at 62.1, 65.9 and $74.5 \mathrm{~cm}^{-1}$ had to be added to the model in order to reproduce the progression of the data curves. Whereas the two modes at 43.5 and $53.9 \mathrm{~cm}^{-1}$ can be fitted well, the model cannot account for the deep dip at $59 \mathrm{~cm}^{-1}$. This behavior is not yet understood but is likely a consequence of the strong deviation of the 9-cis structure from the all-trans configuration. The dip coincides with a strong water vapor absorption line, but since this feature is not observed in the room-temperature spectrum of the isomer we rule out that the dip is related to absorption from residual water vapor in the beam path.

As was shown by model calculations, the different low-frequency resonances originate from collective torsional or skeletal twisting modes with a rather poor degree of localization [8]. Nevertheless these modes are confined to different parts of the molecular chain. By comparing the absorption characteristics of the three different 
Table 1

Molecular parameters extracted from the fit of the $10 \mathrm{~K}$ index and absorption data based on Eq. (1) ${ }^{\mathrm{a}}$

\begin{tabular}{|c|c|c|c|c|c|}
\hline & \multicolumn{2}{|l|}{ All-trans } & \multirow{2}{*}{$\begin{array}{l}\text { 13-cis } \\
\text { This work }^{\mathrm{c}} \\
\end{array}$} & \multirow{2}{*}{$\begin{array}{l}\text { 9-cis } \\
\text { This work }^{\mathrm{d}} \\
\end{array}$} & \multirow[t]{2}{*}{ Assignment } \\
\hline & Ref. [8] & This work $^{\mathrm{b}}$ & & & \\
\hline$v_{1}$ & 46 & $46.6 \pm 0.2$ & $41.7 \pm 0.4$ & $43.5 \pm 0.3$ & $\mathrm{C}_{9}-\mathrm{C}_{14}$ \\
\hline$\Gamma_{1}$ & & $5.2 \pm 0.7$ & $6.6 \pm 0.5$ & $7.6 \pm 0.1$ & $\begin{array}{l}\text { Chain, terminal } \\
\text { part }\end{array}$ \\
\hline$S_{1}$ & & $0.018 \pm 0.003$ & $0.004 \pm 0.001$ & $0.003 \pm 0.001$ & \\
\hline$I_{1}$ & & $1.39 \pm 0.23$ & $0.39 \pm 0.10$ & $0.33 \pm 0.11$ & \\
\hline$v_{2}$ & 53 & $54.4 \pm 0.2$ & $54.8 \pm 0.4$ & $53.9 \pm 0.1$ & $\mathrm{C}_{1}-\mathrm{C}_{6}$ \\
\hline$\Gamma_{2}$ & & $4.7 \pm 0.6$ & $7.6 \pm 0.5$ & $6.2 \pm 0.3$ & Ring \\
\hline$S_{2}$ & & $0.011 \pm 0.002$ & $0.006 \pm 0.001$ & $0.007 \pm 0.001$ & \\
\hline$I_{2}$ & & $1.16 \pm 0.21$ & $1.02 \pm 0.17$ & $1.17 \pm 0.17$ & \\
\hline$v_{3}$ & - & $61.0 \pm 1.3$ & $61.0 \pm 2.0$ & - & \\
\hline$\Gamma_{3}$ & & $2.8 \pm 2.1$ & $5.2 \pm 0.7$ & - & \\
\hline$S_{3}$ & & $0.001 \pm 0.001$ & $0.001 \pm 0.001$ & - & \\
\hline$I_{3}$ & & $0.13 \pm 0.13$ & $0.21 \pm 0.21$ & & \\
\hline$v_{4}$ & 64 & $66.2 \pm 0.7$ & $64.8 \pm 2.8$ & - & $\mathrm{C}_{6}-\mathrm{C}_{9}$ \\
\hline$\Gamma_{4}$ & & $3.4 \pm 1.2$ & $6.9 \pm 1.7$ & - & Chain, middle part \\
\hline$S_{4}$ & & $0.002 \pm 0.001$ & $0.003 \pm 0.001$ & - & \\
\hline$I_{4}$ & & $0.31 \pm 0.16$ & $0.71 \pm 0.24$ & & \\
\hline$v_{5}$ & 68 & $69.3 \pm 1.0$ & $68.6 \pm 6.2$ & - & $\mathrm{C}_{6}-\mathrm{C}_{9}$ \\
\hline$\Gamma_{5}$ & & $2.4 \pm 1.2$ & $7.2 \pm 1.2$ & - & Chain, middle part \\
\hline$S_{5}$ & & $0.001 \pm 0.001$ & $0.002 \pm 0.001$ & - & \\
\hline$I_{5}$ & & $0.17 \pm 0.17$ & $0.53 \pm 0.27$ & & \\
\hline$v_{6}$ & 89 & $90.6 \pm 0.2$ & - & - & \\
\hline$\Gamma_{6}$ & & $4.9 \pm 0.6$ & - & - & \\
\hline$S_{6}$ & & $0.008 \pm 0.001$ & - & - & \\
\hline$I_{6}$ & & $2.33 \pm 0.29$ & & & \\
\hline
\end{tabular}

a The extracted parameters are the resonance frequencies $v_{j}\left(\mathrm{~cm}^{-1}\right)$, linewidths $\Gamma_{j}\left(\mathrm{~cm}^{-1}\right)$, dimensionless oscillator strengths $S_{j}$ and resulting band strengths $I_{j}\left(\mathrm{~km} \mathrm{~mol}^{-1}\right)$. The errors are the S.D. of the fit. The second column lists the results of the FTIR measurement of all-trans retinal at $15 \mathrm{~K}$ in [8]. The last column gives our assignment of the approximate location of the mode in the molecular chain. ${ }^{\mathrm{b}}$ A linear slope of the form $v\left[\mathrm{~cm}^{-1}\right] \times 0.20 \mathrm{M}^{-1}$ was added to the fit of the absorptivity.

${ }^{\mathrm{c}}$ A linear slope of the form $v\left[\mathrm{~cm}^{-1}\right] \times 0.13 \mathrm{M}^{-1}$ was added to the fit of the absorptivity.

${ }^{\mathrm{d}}$ No slope was added to the fit.

isomeric configurations of the retinal molecule we propose an assignment of the approximate localization of these modes in the molecular chain.

Our observation that the feature at $54 \mathrm{~cm}^{-1}$ occurs in all three isomers (see Table 1), independent of the configuration of the polyene chain $\mathrm{C}_{7}-\mathrm{C}_{15}$, implies a localization of this mode at the ring $\mathrm{C}_{1}-\mathrm{C}_{6}$. In contrast the mode at $47 \mathrm{~cm}^{-1}$, which is very distinct in the absorptivity of the alltrans isomer, occurs shifted by 4-5 wavenumbers to lower frequencies and is only weakly active in the 9-cis and 13-cis configurations. This suggests a localization of the mode at the terminal part of the chain $\mathrm{C}_{9}-\mathrm{C}_{14}$. Since the modes at 66 and $69 \mathrm{~cm}^{-1}$ occur equally strong only in the all-trans and 13-cis retinal sample we assign a localization at the $\mathrm{C}$ chain close to the $\mathrm{C}_{9}$ and far from the $\mathrm{C}_{13}$ double bond $\left(\mathrm{C}_{6}-\mathrm{C}_{9}\right)$.

We finally note that our assignment of the localization of the different modes supports the assignment based on density functional calculations for all-trans retinal [8].

\section{Conclusion}

In this work we measured the FIR absorption and dispersion of three different retinal isomers by 
THz-TDS. We observe distinct differences at $10 \mathrm{~K}$ as well as at room temperature among the isomers, demonstrating the feasibility of a femtosecond time-resolved experiment in this interesting spectral region. We extract important parameters of the dynamics of the molecules' low-frequency torsional motion by using a standard expression for Lorentzian oscillators to model the absorption and index data. By comparing the absorption spectra of the three isomers it is possible to deduce the approximate localization of the different vibrational modes within the molecule.

\section{Acknowledgements}

The authors would like to thank B. Schubach, Department of Physical Chemistry, Freiburg, Germany, for guidance in the sample preparation. This work was supported by DFG, under SFB 276, TP C14.

\section{References}

[1] L. Stryer, Biochemistry, fourth ed., Freeman, New York, 1995.

[2] Q. Wang, R.W. Schoenlein, L.A. Peteanu, R.A. Mathies, C.V. Shank, Science 266 (1994) 422.
[3] G. Haran, E.A. Morlino, J. Matthes, R.H. Callender, R.M. Hochstrasser, J. Phys. Chem. A 103 (1999) 2202.

[4] S. Hahn, G. Stock, J. Phys. Chem. B 104 (2000) 1146.

[5] R.W. Schoenlein, L.A. Peteanu, R.A. Mathies, C.V. Shank, Science 254 (1991) 412.

[6] D.L. Rousseau, Optical Techniques in Biological Research, Academic Press, Orlando, 1984.

[7] A. Warshel, M. Karplus, J. Am. Chem. Soc. 96 (1974) 5677.

[8] F.L. Gervasio, G. Cardini, P.R. Salvi, V. Schettino, J. Phys. Chem. A 102 (1998) 2131.

[9] S.W. Lin, M. Groesbeek, I. van der Hoef, P. Verdegem, J. Lugtenburg, R.A. Mathies, J. Phys. Chem. B 102 (1998) 2787.

[10] D.R. Grischkowsky, Ch. Fattinger, M. van Exter, S.R. Keiding, J. Opt. Soc. Am. B 7 (1990) 2006.

[11] A.G. Markelz, A. Roitberg, E.J. Heilweil, Chem. Phys. Lett. 320 (2000) 42.

[12] G. Haran, W.-D. Sun, K. Wynne, R.M. Hochstrasser, Chem. Phys. Lett. 274 (1997) 365.

[13] R. McElroy, K. Wynne, Phys. Rev. Lett. 79 (1997) 3078.

[14] M. Schall, P. Uhd Jepsen, Opt. Lett. 25 (2000) 13.

[15] M. Schall, H. Helm, S.R. Keiding, Int. J. Infrared Millimeter Waves 20 (1999) 595.

[16] M. Walther, Diploma Thesis, University of Freiburg, Germany, 1999.

[17] K.D. Moeller, W.G. Rothschild, Far-infrared Spectroscopy, Wiley, New York, 1971. 